\title{
COMPARISON PRINCIPLES FOR IMPULSIVE PARABOLIC EQUATIONS WITH APPLICATIONS TO MODELS OF SINGLE SPECIES GROWTH
}

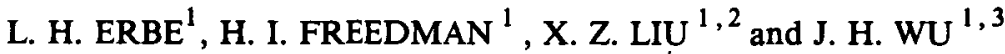

(Received 30 November 1989)

\begin{abstract}
This paper establishes some maximum and comparison principles relative to lower and upper solutions of nonlinear parabolic partial differential equations with impulsive effects. These principles are applied to obtain some sufficient conditions for the global asymptotic stability of a unique positive equilibrium in a reactiondiffusion equation modeling the growth of a single-species population subject to abrupt changes of certain important system parameters.
\end{abstract}

\section{Introduction}

The growth of a population diffusing throughout its habitat is often modeled by a reaction-diffusion equation. Much has been done under the assumption that the system parameters, including those parameters related to the population environment, either are constant or change continuously (see, e.g, [1], [2], [8], [11], [12], [13], [14] and [24]). However, one may easily visualise situations in nature where abrupt changes such as harvesting, disasters and instantaneous stocking may occur. Consequently, we wish to consider such a reaction-diffusion model with impulses.

The qualitative study of impulsive differential equations is rather difficult because of the special features possessed by these systems such as pulse

\footnotetext{
${ }^{1}$ Applied Mathematics Institute, University of Alberta, Edmonton, Canada T6G 2 G1.

${ }^{2}$ Present address: Department of Applied Mathematics, University of Waterloo, Waterloo, Canada.

${ }^{3}$ Present address: Department of Mathematics, York University, North York, Canada.

(C) Copyright Australian Mathematical Society 1991, Serial-fee code 0334-2700/91
} 
phenomena, confluence and the loss of autonomy. Nevertheless, it should be mentioned that the theory of impulsive ordinary differential equations has been considerably developed (see, e.g., [3], [18], [19], [21], [22] and [26]). However, to the best of our knowledge, the corresponding theory for partial differential equations with impulses has not been investigated.

In this paper, we hope to make a start in the study of the dynamics of second-order parabolic equations with impulses. Such equations seem to provide a natural framework for the mathematical modeling of population growth in the case where the population of a given species diffuses in its habitat and is regulated by some impulse factors at certain moments.

One of the objectives of this paper is to establish several maximum and comparison principles for scalar impulsive parabolic partial differential equations in which impulses occur at fixed moments. Our investigation indicates that if the impulses are not too drastic, or if both the reaction rate and the impulse satisfy a global Lipschitz condition, then the classical maximum and comparison principles for parabolic equations without impulses (see, e.g. [15], [16], [17], [23] and [25]) still hold for impulsive parabolic partial differential equations.

The established comparison principle provides a spatially homogeneous estimate of the solution by a maximal and minimal ordinary differential equation subject to impulsive effects. We shall apply this spatially homogeneous estimate and the Liapunov function method to obtain some sufficient conditions for global asymptotic stability of the positive equilibrium of a singlespecies population growth model, where the impulses describe instantaneous harvesting, immigration or disasters. It is shown that increasing the length of time between successive impulses tends to stabilise the system.

This paper is organised as follows. In Section 2, we prove several maximum and comparison principles for general second-order parabolic partial differential equations. In Section 3, we use these results in a model of singlespecies population growth to obtain some global asymptotic stability results of the unique positive equilibrium state.

\section{Maximum principles and comparison techniques}

Let $\Omega$ be a smooth bounded domain in $R^{l}, Q_{T}=(0, T] \times \Omega$ and $\Gamma_{T}=$ $(0, T) \times \partial \Omega$ where $T>0$. Given a partition $0<t_{1}<t_{2}<\cdots<t_{p}<T$ of $[0, T]$, we introduce the following notations

$$
P_{k}\left\{\left(t_{k}, x\right) ; x \in \Omega\right\}, \quad P=\bigcup_{k=1}^{p} P_{k},
$$




$$
\Lambda_{k}=\left\{\left(t_{k}, x\right) ; x \in \partial \Omega\right\}, \quad \Lambda=\bigcup_{k=1}^{p} \Lambda_{k}
$$

and define $C^{1,2}\left(Q_{T}, P\right)$ as the set of all functions $u:[0, T] \times \bar{\Omega} \rightarrow R$ satisfying the following conditions:

(i) $u(t, x)$ is continuously differentiable for $(t, x) \in \bar{Q}_{T} \backslash(P \cup \Lambda)$;

(ii) $u_{x x}(t, x)$ exists and is continuous for $(t, x) \in Q_{T} \backslash P$;

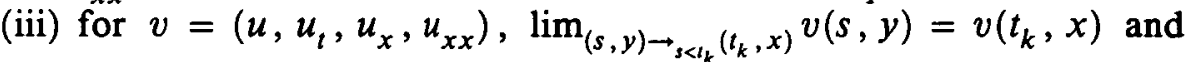
$\lim _{(s, y) \rightarrow>_{s>>_{k}}\left(t_{k}, x\right)} v(s, y)$ exists for $k=1, \ldots, p$ and $x \in \overline{\boldsymbol{\Omega}}$ where $u_{x}=\left(\frac{\partial u}{\partial x_{1}}, \ldots, \frac{\partial u}{\partial x_{l}}\right), u_{x x}=\left(\frac{\partial^{2} u}{\partial x_{1}^{2}}, \frac{\partial^{2} u}{\partial x_{1} \partial x_{2}}, \ldots, \frac{\partial^{2} u}{\partial x_{l}^{2}}\right)$ and $u_{t}=\frac{\partial u}{\partial t}$.

A function $f \in C\left[Q_{T} \times R \times R^{l} \times R^{l^{2}}, R\right]$ is said to be elliptic at a point $(t, x)$ if, for any $u \in R, v \in R^{l}, \gamma=\left(\gamma_{i k}\right), s=\left(s_{i k}\right) \in R^{l^{2}}$, the quadratic form $\sum_{i, k=1}^{l}\left(\gamma_{i k}-s_{i k}\right) \lambda_{i} \lambda_{k} \leq 0$ for an arbitrary vector $\lambda=\left(\lambda_{1}, \ldots, \lambda_{l}\right) \in R^{l}$ implies $f(t, x, u, v, \gamma) \leq f(t, x, u, v, s)$. The differential equation $u_{t}=$ $f\left(t, x, u, u_{x}, u_{x x}\right)$ is called a parabolic equation, if $f$ is elliptic at each point $(t, x) \in Q_{T}$.

We consider the following parabolic equation

$$
u_{t}=f\left(t, x, u, u_{x}, u_{x x}\right), \quad(t, x) \in Q_{T} \backslash P
$$

subject to the initial condition

$$
u(0, x)=u_{0}(x) \quad \text { on } \bar{\Omega},
$$

the boundary condition

$$
B u(t, x)=\varphi(t, x) \quad \text { on } \Gamma_{T} \backslash \Lambda
$$

and the impulse at fixed time $t_{k}, 1 \leq k \leq p$,

$$
u\left(t_{k}^{+}, x\right)-u\left(t_{k}, x\right)=g_{k}\left(u\left(t_{k}, x\right)\right), \quad 1 \leq k \leq p, x \in \bar{\Omega}
$$

where

(i) $g_{k}: R \rightarrow R, 1 \leq k \leq p$, is continuous and the mapping $x+g_{k}(x)$ is increasing for $x \in R$;

(ii) $B: C^{1,2}\left(Q_{T}, P\right) \rightarrow C\left(\Gamma_{T} \backslash \Lambda, R\right)$ is a boundary operator defined by

$$
B u(t, x)=p(t, x) u(t, x)+q(t, x) \frac{\partial}{\partial n} u(t, x) \quad \text { on } \Gamma_{T} \backslash \Lambda
$$

for any $u \in C^{1,2}\left(Q_{T}, P\right)$ where $n$ is an outnormal vector at $(t, x) \in \Gamma_{T}$, and $p$ and $q$ are nonnegative continuous functions with $p(t, x)+q(t, x)>0$ on $\Gamma_{T}$.

A function $u \in C^{1,2}\left(Q_{T}, P\right)$ satisfying (2.1)-(2.4) is called a solution of the initial-boundary-value problem (IBVP for short) (2.1)-(2.4). A function 
$u \in C^{1,2}\left(Q_{T}, P\right)$ is called an upper solution of IBVP (2.1) and (2.4) if it satisfies

$$
\begin{gathered}
u_{t} \geq f\left(t, x, u, u_{x}, u_{x x}\right) \quad \text { on } Q_{T} \backslash P, \\
B u(t, x) \geq \varphi(t, x) \quad \text { on } \Gamma_{T} \backslash \Lambda, \\
u\left(t_{k}^{+}, x\right)-u\left(t_{k}, x\right) \geq g_{k}\left(u\left(t_{k}, x\right)\right), \quad 1 \leq k \leq p, x \in \bar{\Omega} .
\end{gathered}
$$

Lower solutions can be defined analogously by reversing the above inequalities.

We start with a strong version of the classical maximum-value principle.

Theorem 2.1. Assume that $v, w \in C^{1,2}\left(Q_{T}, P\right)$ are given such that

$$
\begin{gathered}
\sup _{Q_{T} \backslash P}\left\{v_{t}-f\left(t, x, v, v_{x}, v_{x x}\right)\right\} \leq 0, \\
v\left(t_{k}^{+}, x\right)-v\left(t_{k}, x\right) \leq g_{k}\left(v\left(t_{k}, x\right)\right), \quad 1 \leq k \leq p, x \in \bar{\Omega}, \\
\inf _{Q_{T} \backslash P}\left\{w_{t}-f\left(t, x, w, w_{x}, w_{x x}\right)\right\} \geq 0, \\
w\left(t_{k}^{+}, x\right)-w\left(t_{k}, x\right) \geq g_{k}\left(w\left(t_{k}, x\right)\right), \quad 1 \leq k \leq p, x \in \bar{\Omega}, \\
v(0, x)<w(0, x), \quad x \in \bar{\Omega}, \\
\sup _{\Gamma_{T} \backslash \Lambda}\{B(t, x)-B w(t, x)\}<0 .
\end{gathered}
$$

Then $v(t, x)<w(t, x)$ on $\bar{Q}_{T}$, if one of the inequalities (2.8) and (2.10) is strict.

Proof. Let $m(t, x)=w(t, x)-v(t, x)$. If the conclusion is not true, then there exists a $\tau>0$ and $y \in \bar{\Omega}$, such that one of the following three cases holds:

Case 1: $\tau \neq t_{k}, 1 \leq k \leq p, m(t, x)>0$ on $(0, \tau) \times \bar{\Omega}$ and $m(\tau, y)=0$;

Case 2: $\tau=t_{k}$ for some $k, 1 \leq k \leq p, m(t, x)>0$ on $\left[0, t_{k}\right) \times \bar{\Omega}$ and $m\left(t_{k}, y\right)=0$;

Case 3: $\tau=t_{k}$ for some $k, 1 \leq k \leq p, m(t, x)>0$ on $\left[0, t_{k}\right] \times \bar{\Omega}$ and $m\left(t_{k}^{+}, y\right) \leq 0$.

In the first case, $m(t, x) \geq 0$ on $[0, \tau] \times \bar{\Omega}$ by continuity. Therefore, if $(\tau, y) \in \Gamma_{T}$, then

$$
\frac{\partial}{\partial n} m(\tau, y)=\lim _{h \rightarrow 0^{+}} \frac{1}{h}[m(\tau, y)-m(\tau, y-h n)] \leq 0,
$$

which leads to the following contradiction

$$
0<B m(\tau, y)=q(\tau, y) \frac{\partial}{\partial n} m(\tau, y) \leq 0 .
$$


Hence $(\tau, y) \in Q_{T}$. Evidently, $m(\tau, x)$ attains its minimum at $(\tau, y)$. This implies $m_{x_{i}}(\tau, y)=0$ for $1 \leq i \leq l$ and the quadratic form

$$
\sum_{i, k=1}^{l} m_{x_{i} x_{k}}(\tau, y) \lambda_{i} \lambda_{k} \geq 0
$$

for arbitrary vector $\lambda \in R^{l}$. This implies that $v(\tau, y)=w(\tau, y), v_{x}(\tau, y)=$ $w_{x}(\tau, y)$ and $\sum_{i, k=1}^{l}\left(\partial^{2} / \partial x_{i} \partial x_{k}\right)[v(\tau, y)-w(\tau, y)] \lambda_{i} \lambda_{k} \leq 0$. From the ellipticity of $f$, it follows that

$$
\begin{aligned}
f\left(\tau, y, w(\tau, y), w_{x}(\tau, y), w_{x x}(\tau, y)\right) \\
\geq f\left(\tau, y, v(\tau, y), v_{x}(\tau, y), v_{x x}(\tau, y)\right) .
\end{aligned}
$$

Therefore if one of the inequalities (2.8) and (2.10) is strict, then

$$
\begin{aligned}
m_{t}(\tau, y)> & f\left(\tau, y, w(\tau, y), w_{x}(\tau, y), w_{x x}(\tau, y)\right) \\
& -f\left(\tau, y, v(\tau, y), v_{x}(\tau, y), v_{x x}(\tau, y)\right) \geq 0 .
\end{aligned}
$$

However, $(\tau-h, y) \in Q_{T}$ for sufficiently small $h>0$. This implies $m(\tau-h, y)>m(\tau, y)=0$, and thus $m_{t}(\tau, y) \leq 0$ which is a contradiction of (2.14).

In Case 2, we have $m_{t}\left(t_{k}, y\right)=\lim _{s \rightarrow t_{k}} m_{t}(s, y) \leq 0$, for otherwise there exists $\delta>0$ such that $m_{t}\left(t_{k}, y\right) \geq \delta$ and consequently $m_{t}\left(t_{k}-h, y\right) \geq \frac{\delta}{2}$ for sufficiently small $h>0$. Thus for $\eta>0$ small enough,

$$
m\left(t_{k}-h, y\right)-m\left(t_{k}-h-\eta, y\right) \geq \delta \eta / 2 \text {. }
$$

Letting $h \rightarrow 0$ in the above inequality, we get $-m\left(t_{k}-\eta, y\right) \geq \delta \eta / 2>$ 0 , which is contrary to the assumption that $m(t, x)>0$ on $\left[0, t_{k}\right) \times \bar{\Omega}$. Therefore $m_{t}\left(t_{k}, y\right) \leq 0$. We can employ the same argument as for Case 1 to prove that

$$
\frac{\partial}{\partial n} m\left(t_{k}, y\right)=\lim _{s \rightarrow t_{k}^{-}} \frac{\partial}{\partial n} m(s, y) \leq 0 .
$$

From (2.13) and the left continuity of $m(t, x)$, we get

$$
B m\left(t_{k}, x\right)=B w\left(t_{k}, x\right)-B v\left(t_{k}, x\right)>0, \quad x \in \bar{\Omega} .
$$

Therefore by using the same argument as for Case 1 , we can show $y \in \Omega$. Moreover, by (2.8) and (2.10), we get

$$
\begin{gathered}
v_{t}\left(t_{k}, y\right) \leq f\left(t, y, v\left(t_{k}, y\right), v_{x}\left(t_{k}, y\right), v_{x x}\left(t_{k}, y\right)\right), \\
w_{t}\left(t_{k}, y\right) \geq f\left(t, y, w\left(t_{k}, y\right), w_{x}\left(t_{k}, y\right), w_{x x}\left(t_{k}, y\right)\right),
\end{gathered}
$$

and one of the inequalities (2.16) and (2.17) is strict. We can now use the same argument as for Case 1 to derive $m_{t}\left(t_{k}, y\right)>0$ which is a contradiction of our earlier conclusion that $m_{t}\left(t_{k}, y\right) \leq 0$. 
In Case 3, we have $0 \geq m\left(t_{k}^{+}, x\right) \geq w\left(t_{k}, y\right)+g_{k}\left(w\left(t_{k}, y\right)\right)-v\left(t_{k}, y\right)-$ $g_{k}\left(v\left(t_{k}, y\right)\right)$ which implies $w\left(t_{k}, y\right) \leq v\left(t_{k}, y\right)$ since $I+g_{k}$ is increasing. This is contrary to the assumption that $m(t, x)>0$ on $\left[0, t_{k}\right] \times \bar{\Omega}$. The proof is then complete.

To dispense with the strict inequalities required in the above theorem, we introduce the following assumption:

(H1) there exists a function $z \in C^{1,2}\left(Q_{T}, P\right)$ such that

$$
\inf _{Q_{T} \backslash P} z(t, x)>0, \quad \inf _{\Gamma_{T} \backslash \Lambda} \frac{\partial}{\partial n} z(t, x)>0
$$

and for sufficiently small $\varepsilon>0$ and any given $u \in C^{1,2}\left(Q_{T}, P\right)$, we have

$$
\begin{gathered}
\inf _{Q_{T} \backslash P}\left\{\varepsilon z_{t}-f\left(t, x, u+\varepsilon z, u_{x}+\varepsilon z_{x}, u_{x x}+\varepsilon z_{x x}\right)+f\left(t, x, u, u_{x}, u_{x x}\right)\right\}>0, \\
g_{k}\left(u\left(t_{k}, x\right)+\varepsilon z\left(t_{k}, x\right)\right)-g_{k}\left(u\left(t_{k}, x\right)\right) \leq \varepsilon\left[z\left(t_{k}^{+}, x\right)-z\left(t_{k}, x\right)\right], \\
1 \leq k \leq p .
\end{gathered}
$$

THEOREM 2.2. Suppose that (H1) holds and $v$ and $w$ are lower and upper solutions of IBVP (2.1), (2.2), (2.4), respectively. Then the relations

$$
\begin{gathered}
v(0, x) \leq w(0, x) \quad \text { for } x \in \bar{\Omega}, \\
B v(t, x) \leq B w(t, x) \quad \text { on } \Gamma_{T} \backslash \Lambda
\end{gathered}
$$

imply $v(t, x) \leq w(t, x)$ on $\bar{Q}_{T}$.

Proof. We consider $\tilde{w}=w+\varepsilon z$. Clearly $\tilde{w} \in C^{1,2}\left(Q_{T}, P\right)$. By (2.18), we have

$$
\begin{aligned}
& \inf _{Q_{T} \backslash P}\left\{\tilde{w}_{t}-f\left(t, x, \tilde{w}, \tilde{w}_{x}, \tilde{w}_{x x}\right)\right\} \\
& \quad=\inf _{Q_{T} \backslash P}\left\{w_{t}+\varepsilon z_{t}-f\left(t, x, w+\varepsilon z, w_{x}+\varepsilon z_{x}, w_{x x}+\varepsilon z_{x x}\right)\right\} \\
& \geq \inf _{Q_{T} \backslash P}\left\{w_{t}-f\left(t, x, w, w_{x}, w_{x x}\right)\right\} \\
& \quad+\inf _{Q_{T} \backslash P}\left\{f\left(t, x, w, w_{x}, w_{x x}\right)\right. \\
& \left.\quad \quad-f\left(t, x, w+\varepsilon z, w_{x}+\varepsilon z_{x}, w_{x x}+\varepsilon z_{x x}\right)+\varepsilon z_{t}\right\} \\
& \quad+\inf _{Q_{T} \backslash P}\left\{\varepsilon z_{t}-f\left(t, x, w+\varepsilon z, w_{x}+\varepsilon z_{x}, w_{x x}+\varepsilon z_{x x}\right)\right. \\
& \left.\quad+f\left(t, x, w, w_{x}, w_{x x}\right)\right\} \\
& >0 .
\end{aligned}
$$


Evidently, $v(0, x) \leq w(0, x)<\tilde{w}(0, x)$ on $\bar{\Omega}$, and

$$
\begin{aligned}
\sup _{\Gamma_{T} \backslash \Lambda}\{B v(t, x)-B \tilde{w}(t, x)\} & =\sup _{\Gamma_{T} \backslash \Lambda}\{B v(t, x)-B w(t, x)-\varepsilon B z(t, x)\} \\
& \leq-\varepsilon \inf _{\Gamma_{T} \backslash \Lambda}\left\{p(t, x) z(t, x)+q(t, x) \frac{\partial}{\partial n} z(t, x)\right\} \\
& <0 .
\end{aligned}
$$

Moreover, by (2.19) we have

$$
\begin{aligned}
\tilde{w}\left(t_{k}^{+}, x\right)=w\left(t_{k}^{+}, x\right) & +\varepsilon z\left(t_{k}^{+}, x\right) \\
\geq w\left(t_{k}, x\right) & +g_{k}\left(w\left(t_{k}, x\right)\right)+\varepsilon z\left(t_{k}^{+}, x\right) \\
\geq w\left(t_{k}, x\right) & +g_{k}\left(w\left(t_{k}, x\right)+\varepsilon z\left(t_{k}, x\right)\right) \\
& +g_{k}\left(w\left(t_{k}, x\right)+\varepsilon z\left(t_{k}, x\right)\right)-g_{k}\left(w\left(t_{k}, x\right)\right) \\
= & \tilde{w}\left(t_{k}, x\right)+g_{k}\left(\tilde{w}\left(t_{k}, x\right)\right) .
\end{aligned}
$$

Therefore $v$ and $\tilde{w}$ satisfies all assumptions of Theorem 2.1. This implies $v(t, x)<\tilde{w}(t, x)=w(t, x)+\varepsilon z(t, x)$. Hence $v(t, x) \leq w(t, x)$ follows by taking the limit as $\varepsilon \rightarrow 0$. A similar argument also leads to $v(t, x) \leq w(t, x)$ if (2.20) and (2.21) hold. The proof is then complete.

Employing a similar argument, we obtain the following comparison principle.

THEOREM 2.3. Assume that $u, v \in C^{1,2}\left(Q_{T}, P\right)$ satisfy (2.8)-(2.13). Moreover, suppose that there exist functions $G: \bar{Q}_{T} \times[0, \infty) \times[0, \infty) \times[0, \infty) \rightarrow R$, and $z \in C^{1,2}\left(Q_{T}, P\right)$ such that

(i) $\inf _{Q_{T} \backslash P} z(t, x)>0, \inf _{\Gamma_{T} \backslash \Lambda} \frac{\partial}{\partial n} z(t, x)>0$,

(ii) whenever $\bar{u}, \underline{u} \in C^{1,2}\left(Q_{T}, P\right)$ and $\bar{u} \leq \underline{u}$;

$$
\begin{aligned}
& f\left(t, x, \bar{u}, \bar{u}_{x}, \bar{u}_{x x}\right)-f\left(t, x, \underline{u}, \underline{u}_{x}, \underline{u}_{x x}\right) \\
& \quad \leq G\left(t, x, \bar{u}-\underline{u},\left|\bar{u}_{x}-\underline{u}_{x}\right|,\left|\bar{u}_{x x}-\underline{u}_{x x}\right|\right)
\end{aligned}
$$

(iii) $\inf _{Q_{T} \backslash P}\left\{\varepsilon z_{t}-G\left(t, x, \varepsilon z, \varepsilon z_{x}, \varepsilon z_{x x}\right)\right\}>0$ for sufficiently small $\varepsilon>0$. Then for any solution $u(t, x)$ of $(2.1)-(2.4)$ with

$$
v(0, x) \leq u_{0}(x) \leq w(0, x) \text { on } \bar{\Omega}
$$

and

we have

$$
B v \leq B u \leq B w \text { on } \Gamma_{T} \backslash \Lambda
$$

$$
v(t, x) \leq u(t, x) \leq w(t, x) \quad \text { on } \bar{Q}_{T} .
$$

REMARK 2.1. If $g_{k}, 1 \leq k \leq p$, satisfies the one-sided Lipschitz condition

$$
g_{k}(u)-g_{k}(v) \leq \alpha(u-v) \quad \text { for } u \geq v, 1 \leq k \leq p,
$$


where $\alpha \in[0,1)$ is a constant, and $B$ is the Riemann boundary operator (that is, $q(t, x)=0$ ), then the assumption (H1) in Theorem 2.2 can be replaced by a weaker hypothesis, namely, a one-sided Lipschitz condition of the form

$$
f(t, x, u, r, s)-f(t, x, v, r, s) \leq L(u-v)
$$

for all $(t, x) \in \bar{Q}_{T}, u, v \in R, r \in R^{l}, s \in R^{l^{2}}$ and $u>v$.

Proof. Define $\tilde{w} \in C^{1,2}\left(Q_{T}, P\right)$ by

$$
\tilde{w}(t, x)=w(t, x)+\varepsilon G(k) e^{2 L t}
$$

for $(t, x) \in[0, T] \times \bar{\Omega}$ and $t_{k-1}<t \leq t_{k}, 1 \leq k \leq p+1$ where $t_{0}=0$, $t_{p+1}=T$ and

$$
G(0)=1, \quad G(k)=\frac{1}{(1-\alpha)^{k}} \quad \text { for } 1 \leq k \leq p+1 .
$$

Then by (2.10) and (2.21), in the interval $\left(t_{k-1}, t_{k}\right)$ we have

$$
\begin{aligned}
\tilde{w}_{t}-f(t, & \left.x, \tilde{w}, \tilde{w}_{x}, \tilde{w}_{x x}\right) \\
& =w_{t}+2 L \varepsilon G(k) e^{2 L t}-f\left(t, x, \tilde{w}, \tilde{w}_{x}, \tilde{w}_{x x}\right) \\
& \geq f\left(t, x, w, \tilde{w}_{x}, \tilde{w}_{x x}\right)+2 L \varepsilon G(k) e^{2 L t}-f\left(t, x, \tilde{w}, \tilde{w}_{x}, \tilde{w}_{x x}\right) \\
& \geq L \varepsilon G(k) e^{2 L t} \\
& \geq L \varepsilon,
\end{aligned}
$$

that is,

$$
\inf _{Q_{T} \backslash P}\left\{\tilde{w}_{t}-f\left(t, x, \tilde{w}, \tilde{w}_{x}, \tilde{w}_{x x}\right)\right\}>0 .
$$

Moreover, for $1 \leq k \leq p$, we have

$$
\begin{aligned}
& \tilde{w}\left(t_{k}^{+}, x\right)-\tilde{w}\left(t_{k}, x\right) \\
& \quad=w\left(t_{k}^{+}, x\right)-w\left(t_{k}, x\right)+\varepsilon[G(k+1)-G(k)] e^{2 L t_{k}} \\
& \quad=g_{k}\left(w\left(t_{k}, x\right)\right)+\varepsilon[G(k+1)-G(k)] e^{2 L t_{k}} \\
& \quad=g_{k}\left(\tilde{w}\left(t_{k}, x\right)\right)+g_{k}\left(w\left(t_{k}, x\right)\right)-g_{k}\left(\tilde{w}\left(t_{k}, x\right)\right)+\varepsilon[G(k+1)-G(k)] e^{2 L t_{k}} \\
& \quad \geq g_{k}\left(\tilde{w}\left(t_{k}, x\right)\right)-\alpha\left[\tilde{w}\left(t_{k}, x\right)-w\left(t_{k}, x\right)\right]+\varepsilon[G(k+1)-G(k)] e^{2 L t_{k}} \\
& \quad \geq g_{k}\left(\tilde{w}\left(t_{k}, x\right)\right)-\alpha \varepsilon G(k+1) e^{2 L t_{k}}+\varepsilon[G(k+1)-G(k)] e^{2 L t_{k}} \\
& \quad \geq g_{k}\left(\tilde{w}\left(t_{k}, x\right)\right),
\end{aligned}
$$

since

$$
-\alpha G(k+1)+G(k+1)-G(k)=0
$$

by definition of $G(k)$. Therefore our conclusion follows from Theorem 2.1. 
REMARK 2.2. Suppose that $f$ and $g_{k}, 1 \leq k \leq p$, satisfy the Lipschitz condition

(H2): there exist positive constants $L_{i}, 1 \leq i \leq 4$, such that

$$
\begin{aligned}
& g_{k}(u)-g_{k}(\bar{u}) \leq L_{1}(u-\bar{u}), \quad 1 \leq k \leq p, \\
& f(t, x, u, r, s)-f(t, x, \bar{u}, \bar{r}, \bar{s}) \leq L_{2}(u-\bar{u})+L_{3}|r-\bar{r}|+L_{4}|s-\bar{s}|
\end{aligned}
$$

for any $u, \bar{u} \in R$, with $u>\bar{u}, r, \bar{r} \in R^{l}$ and $s, \bar{s} \in R^{l^{2}}$.

Moreover, assume that the boundary $\Gamma_{T}$ is regular, that is, there exists a function $h \in C^{2}(\bar{\Omega})$ such that $h(x) \geq 0, \frac{\partial}{\partial n} h(x) \geq 1$ on $\Gamma_{T}$. Then (H1) holds.

In fact, defining $H(x)=e^{L_{1} h(x)}$, we can then choose a constant $N>0$ so that

$$
N>2 L_{2}\left(1+L_{1}\right)^{p}+L_{3}\left|H_{x}\right|+L_{4}\left|H_{x x}\right| \text { on } \bar{Q}_{t}
$$

and

$$
z(t, x)=e^{N T} H(x)+\left(1+L_{1}\right)^{k}+\left[\left(1+L_{1}\right)^{k}-1\right] e^{N T} H^{*}
$$

for $t_{k-1}<t \leq t_{k}, 1 \leq k \leq p+1, x \in \bar{\Omega}$, where $t_{0}=0, t_{p+1}=T$ and $h^{*}=\sup _{x \in \bar{\Omega}} H(x)$. Then for any $u \in C^{1,2}\left(Q_{T}, P\right)$, we have

$$
\begin{aligned}
g_{k}\left(u\left(t_{k}, x\right)+\varepsilon z\left(t_{k}, x\right)\right)- & g_{k}\left(u\left(t_{k}, x\right)\right) \\
\leq & \varepsilon L_{1} z\left(t_{k}, x\right) \\
& \leq \varepsilon L_{1}\left\{e^{N t_{k}} H^{*}+\left(1+L_{1}\right)^{k}+\left[\left(1+L_{1}\right)^{k}-1\right] e^{N T} H^{*}\right\} \\
& =\varepsilon L_{1}\left(1+e^{N T} H^{*}\right)\left(1+L_{1}\right)^{k}
\end{aligned}
$$

Therefore

$$
g_{k}\left(u\left(t_{k}, x\right)+\varepsilon z\left(t_{k}, x\right)\right)-g_{k}\left(u\left(t_{k}, x\right) \leq \varepsilon\left[z\left(t_{k}^{+}, x\right)-z\left(t_{k}, x\right)\right]\right.
$$


Moreover, for $t_{k-1}<t \leq t_{k}$, we have

$$
\begin{aligned}
f(t, & \left.x, u+\varepsilon z, u_{x}+\varepsilon z_{x}, u_{x x}+\varepsilon z_{x x}\right)-f\left(t, x, u, u_{x}, u_{x x}\right) \\
\leq & {\left[L_{4}\left|z_{x x}\right|+L_{3}\left|z_{x}\right|+L_{2} z\right] \varepsilon } \\
\leq & \left\{L_{4} e^{N T}\left|H_{x x}\right|+L_{3} e^{N T}\left|H_{x}\right|+L_{2} e^{N T}|H|\right. \\
& \left.+L_{2}\left(1+L_{1}\right)^{k}+L_{2}\left[\left(1+L_{1}\right)^{k}-1\right] e^{N T} H^{*}\right\} \varepsilon \\
\leq & \left\{L_{4}\left|H_{x x}\right|+L_{3}\left|H_{x}\right|+L_{2}|H|+L_{2}\left(1+L_{1}\right)^{p}\right. \\
& \left.+L_{2}\left[\left(1+L_{1}\right)^{p}-1\right] H^{*}\right\} e^{N T} \varepsilon \\
\leq & {\left[L_{4}\left|H_{x x}\right|+L_{3}\left|H_{x}\right|+2 L_{2}\left(1+L_{1}\right)^{p}\right] e^{N T} \varepsilon . }
\end{aligned}
$$

Hence, by the choice of $N$, we get

$$
\begin{aligned}
& \inf _{\bar{Q}_{T} \backslash P}\left\{\varepsilon z_{t}-f\left(t, x, u+\varepsilon z, u_{x}+\varepsilon z_{x}, u_{x x}+\varepsilon z_{x x}\right)+f\left(t, x, u, u_{x}, u_{x x}\right)\right\} \\
& \quad \geq \inf _{\bar{Q}_{T}}\left\{N-\left[2 L_{2}\left(1+L_{1}\right)^{p}+L_{3}\left|H_{x}\right|+L_{4}\left|H_{x x}\right|\right]\right\} e^{N T} \varepsilon>0 .
\end{aligned}
$$

Therefore (2.18) holds. This verifies (H1).

As an immediate consequence of Theorem 2.2, we get the following invariance result.

TheOREM 2.4. Assume (H1) holds. Moreover, assume that $f(t, x, a, 0,0) \geq$ 0 (or $f(t, x, b, 0,0) \leq 0$ ) and $g_{k}(a) \geq 0, k=1,2, \ldots, p$. Then the closed set $\bar{U}=\{u \in R ; u \geq a\}$ (or $\bar{U}=\{u \in R ; u \leq b\}$, is positively invariant relative to $I B V P(2.1)-(2.4)$. That is, $u_{0}(x) \geq a\left(u_{0}(x) \leq b\right)$ on $\bar{\Omega}$ and $B u(t, x) \geq p(t, x) a$ (or $B u(t, x) \leq p(t, x) b)$ on $\Gamma_{T} \backslash \Lambda$ imply $u(t, x) \geq a($ or $u(t, x) \leq b)$ on $\overline{Q_{T}}$, where $u(t, x)$ is a solution of IBVP (2.1)-(2.4).

An important application of invariance results is the following result about upper bounds and lower bounds obtained by using dominating ordinary differential equations.

THEOREM 2.5. Assume that

(i) (H1) holds,

(ii) $f_{1}, f_{2} \in C([0, T] \times R, R)$ such that

$$
f_{2}(t, u) \leq f(t, x, u, 0,0) \leq f_{1}(t, u) \quad \text { for all }(t, x, u) \in \bar{Q}_{T} \times R \text {, }
$$

(iii) $\gamma(t)$ and $\rho(t)$ are solutions of

$$
\left\{\begin{array}{l}
\dot{\gamma}=f_{1}(t, \gamma), \quad t \neq 0, t_{k}, T, \quad 1 \leq k \leq p, \\
\gamma\left(t_{k}^{+}\right)-\gamma\left(t_{k}\right)=g_{k}\left(\gamma\left(t_{k}\right)\right), \quad 1 \leq k \leq p, \\
\gamma(0)=\gamma_{0}
\end{array}\right.
$$


and

$$
\left\{\begin{array}{l}
\dot{\rho}=f_{2}(t, \rho), \quad t \neq 0, t_{k}, T, \quad 1 \leq k \leq p, \\
\rho\left(t_{k}^{+}\right)-\rho\left(t_{k}\right)=g_{k}\left(\rho\left(t_{k}\right)\right), \quad 1 \leq k \leq p \\
\rho(0)=\rho_{0},
\end{array}\right.
$$

(iv) $\rho_{0} \leq u_{0}(x) \leq \gamma_{0}$ on $\bar{\Omega}$

$$
p(t, x) \rho(t) \leq B u(t, x) \leq \rho(t, x) \gamma(t) \quad \text { on } \Gamma_{T} \backslash \Lambda \text {. }
$$

Then $\rho(t) \leq u(t, x) \leq \gamma(t)$ on $\overline{Q_{T}}$, where $u(t, x)$ is the solution of IBVP (2.1)-(2.4).

Proof. Letting

$$
m(t, x)=u(t, x)-\gamma(t)
$$

we have

$$
\begin{gathered}
m_{t}=F\left(t, x, m, m_{x}, m_{x x}\right) \\
m(0, x)=u_{0}(x)-\gamma(0)=u_{0}(x)-\gamma_{0} \leq 0 \quad \text { on } \bar{\Omega} \\
B m(t, x)=B u(t, x)-p(t, x) \gamma(t) \leq 0 \quad \text { on } \Gamma_{T} \backslash \Lambda \\
m\left(t_{k}^{+}, x\right)-m\left(t_{k}, x\right)=G_{k}\left(m\left(t_{k}, x\right)\right), \quad 1 \leq k \leq p
\end{gathered}
$$

where

$$
F\left(t, x, m, m_{x}, m_{x x}\right)=f\left(t, x, m+\gamma, m_{x}, m_{x x}\right)-f_{1}(t, \gamma)
$$

and

$$
G_{k}(m)=g_{k}\left(m+\gamma\left(t_{k}\right)\right)-g_{k}\left(\gamma\left(t_{k}\right)\right) .
$$

According to (ii), we have

$$
F(t, x, 0,0,0)=f(t, x, \gamma, 0,0)-f_{1}(t, \gamma) \leq 0
$$

and by (2.10) we obtain

$$
\begin{aligned}
& \inf _{Q_{T} \backslash P}\left\{\varepsilon z_{t}+F\left(t, x, m-\varepsilon z, m_{x}-\varepsilon z_{x}, m_{x x}-\varepsilon z_{x x}\right)\right.\left.-F\left(t, x, m, m_{x}, m_{x x}\right)\right\} \\
&= \inf _{Q_{r} \backslash P}\left\{\varepsilon z_{t}+f\left(t, x, m-\varepsilon z+\gamma, m_{x}-\varepsilon z_{x}, m_{x x}-\varepsilon z_{x x}\right)-f_{1}(t, \gamma)\right. \\
&=\left.-f\left(t, x, m+\gamma, m_{x}, m_{x x}\right)+f_{1}(t, \gamma)\right\} \\
&=\inf _{Q_{T} \backslash P}\left\{\varepsilon z_{t}-f\left(t, x, m+\gamma, m_{x}+\gamma_{x}, m_{x x}+\gamma_{x x}\right)\right. \\
&\left.\quad+f\left(t, x, m+\gamma-\varepsilon z, m_{x}+\gamma_{x}-\varepsilon z_{x}, m_{x x}+\gamma_{x x}-\varepsilon z_{x x}\right)\right\}
\end{aligned}
$$

$\geq 0$. 
Moreover, we have

$$
\begin{aligned}
& G_{k}\left(u\left(t_{k}, x\right)\right)-G_{k}\left(u\left(t_{k}, x\right)-\varepsilon z\left(t_{k}, x\right)\right) \\
&= g_{k}\left(u\left(t_{k}, x\right)+\gamma\left(t_{k}\right)\right)-g_{k}\left(\gamma\left(t_{k}\right)\right) \\
&-g_{k}\left(u\left(t_{k}, x\right)-\varepsilon z\left(t_{k}, x\right)+\gamma\left(t_{k}\right)\right)+g_{k}\left(\gamma\left(t_{k}\right)\right) \\
& \leq \varepsilon\left[z\left(t_{k}^{+}, x\right)-z\left(t_{k}, x\right)\right] .
\end{aligned}
$$

Therefore $F$ and $G_{k}$ satisfy (H1). By Theorem 2.4, we have $m(t, x) \leq 0$ on $\overline{Q_{T}}$, that is, $u(t, x) \leq \gamma(t)$ on $\overline{Q_{T}}$. Similarly, we can prove that $u(t, x) \geq \rho(t)$ on $\overline{Q_{T}}$. The proof is completed.

\section{Applications to single-species models}

We consider the following reaction-diffusion equation

$$
u_{t}=\alpha \Delta u+u f(u)+c, \quad t \neq t_{k}, k=1,2, \ldots, x \in \Omega
$$

subject to the initial condition

$$
u(0, x)=u_{0}(x) \text { on } \bar{\Omega},
$$

the no-flux boundary condition

$$
\frac{\partial}{\partial n} u(t, x)=0, \quad t \neq t_{k}, k=1,2, \ldots, x \in \partial \Omega,
$$

and the impulses at fixed moments $t_{k}, k=1,2, \ldots$

$$
u\left(t_{k}^{+}, x\right)-u\left(t_{k}, x\right)=g_{k}\left(u\left(t_{k}, x\right)\right), \quad k=1,2, \ldots, x \in \bar{\Omega},
$$

where $u(t, x)$ is the population density at the point $x \in \bar{\Omega}$ and time $t \geq 0$, $f(u)$ is the specific growth rate of $u$ and satisfies standard assumptions [9], [10], $\alpha>0$ is the diffusion coefficient, $g_{k}:[0, \infty) \rightarrow R$ is continuous, $c \geq 0$ is a constant, $\Omega$ is a suitable open and bounded domain, and $\left\{t_{k}\right\}$ is an increasing unbounded sequence.

We consider two cases, $I: c=0, I I: c>0$. Each of these cases can be interpreted biologically. For example, the case where $c=0$, but $g_{k}\left(u\left(t_{k}, x\right)\right)<$ 0 models the growth dynamics in a closed environment with instantaneous harvesting at times $t_{k}, k=1,2, \ldots$, (see [4], [5], [6], [9] for continuous harvesting). On the other hand, the case $c>0$ could be thought of as a model of urban growth of human populations with a constant influx rate of population into the urban environment and occasional rapid changes in population due to heavy immigration $\left(g_{k}>0\right)$ or a disaster (disease, war, etc.), $\left(g_{k}<0\right)$. In this last case, the diffusion is particularly significant in the scenario of a city with expanding boundaries (see [6] for a model with constant stocking). 
We first obtain some results in Case $I: c=0$. The following theorem gives criteria for the global asymptotic stability of the steady state solution, which is an equilibrium representing the carrying capacity of the environment.

\section{THEOREM 3.1. Assume that}

(i) $f(u)$ is continuously differentiable, $\frac{d}{d u}[u f(u)]$ is uniformly bounded for $u \geq 0$ and there exists a constant $K>0$ such that

$$
\begin{gathered}
(u-K) u f(u) \leq-r(u-K)^{2} \quad \text { for } u \geq K, \\
(u-K) u f(u)<0 \quad \text { for } 0<u<K,
\end{gathered}
$$

where $r>0$ is a constant,

(ii) $g_{k}:[0, \infty) \rightarrow(-\infty,+\infty)$ is a globally Lipschitz continuous function such that $x+g_{k}(x)$ is increasing and

$$
\begin{gathered}
x=g_{k}(x) \geq K \quad \text { if } x \geq K, \\
x \leq x+g_{k}(x)<K \quad \text { if } 0<x<K, k=1,2, \ldots,
\end{gathered}
$$

(iii) for any $z>0$,

$$
r\left(t_{k}-t_{k-1}\right)-2 \ln \left(1+\frac{g_{k}(z-K)}{z}\right) \geq \xi_{k} \geq 0, \quad k \geq 2,
$$

where $\xi_{k}$ is a constant, $k \geq 2$,

(iv) the series $\sum_{k=1}^{\infty} \xi_{k}$ is divergent.

Then the solution $x=K$ is globally asymptotically stable, that is, for any $u_{0} \epsilon$ $C(\bar{\Omega},(0, \infty))$ and any solution of $(3.1)-(3.4)$ with $c=0, \lim _{t \rightarrow \infty} u(t, x)=$ $K$ for $x \in \bar{\Omega}$.

Proof. Let $\underline{u}=\min _{x \in \bar{\Omega}} u_{0}(x)$ and $\bar{u}=\max _{x \in \bar{\Omega}} u_{0}(x)$. By Theorem 2.5 and Remark 2.2 one gets $\rho(t) \leqq u(t, x) \leq \gamma(t)$, where $\rho(t)$ and $\gamma(t)$ are solutions of the following impulsive ordinary differential equation

$$
\left\{\begin{array}{l}
\dot{z}=z f(z), \quad t \neq 0, t_{k}, \\
z\left(t_{k}^{+}\right)-z\left(t_{k}\right)=g_{k}\left(z\left(t_{k}\right)\right), \quad k=1,2, \ldots,
\end{array}\right.
$$

subject to the initial conditions

$$
\begin{aligned}
& z(0)=\underline{u} \\
& z(0)=\bar{u},
\end{aligned}
$$

respectively. Therefore to prove the theorem it suffices to prove that for any given $z(0)>0$ the solution of (3.5) tends to $K$ as $t \rightarrow \infty$. Hence, let $z(t)$ be a solution of (3.5) subject to $z(0)>0$. Define $m(t)=(z(t)-K)^{2}$. We distinguish three cases: 
Case 1: $0<z(0)<K$,

Case 2: $z(0)>K$,

Case 3: $z(0)=K$.

If $0<z(0)<K$, it follows from (i) and (ii) that

$$
\begin{gathered}
(z(t)-K)(z(0)-K) \geq 0 \quad \text { for } t \geq 0, \\
m^{\prime}(t)=-(K-z(t)) z(t) f(z(t))<0 \quad \text { for } t \geq 0, t \neq t_{k},
\end{gathered}
$$

and $m\left(t_{k}^{+}\right) \leq m\left(t_{k}\right), k=1,2, \ldots$ Clearly, for any given $\varepsilon>0$, if

$$
0<K-z(0)<\frac{\varepsilon}{1+\varepsilon},
$$

then (3.8) and (3.9) imply that

$$
0 \leq K-z(t) \leq \frac{\varepsilon}{1+\varepsilon}<\varepsilon \quad \text { for } t \geq 0
$$

We claim that for any $z(0)$ with $0<z(0)<K, \lim _{t \rightarrow \infty} z(t)=K$. Otherwise, we can find a constant $\sigma>0$ such that $z(t) \leq K-\sigma$ for $t \geq 0$. Thus from (3.8) we derive

$$
m(t) \leq m(0)-\sigma z(0) \min _{z(0) \leq u \leq k-\sigma} f(u) t<0
$$

as $t \rightarrow \infty$ which is a contradiction to $m(t) \geq 0$.

If $z(0)>K$, from conditions (i), (ii) and (3.8) we get

$$
m^{\prime}(t) \leq-r m(t) \text { for } t \geq 0 \text { and } t \neq t_{k}, 1 \leq k \leq \infty,
$$

and

$$
m\left(t_{k}^{+}\right) \leq m\left(t_{k}\right)+2 \sqrt{m\left(t_{k}\right)} g\left(\sqrt{m\left(t_{k}\right)}+K\right)+g_{k}^{2}\left(\sqrt{m\left(t_{k}\right)}+k\right) .
$$

Define

$$
\psi(u)=u+2 \sqrt{u} g_{k}(\sqrt{u}+K)+g_{k}^{2}(\sqrt{u}+K) .
$$

Clearly $\psi(u)$ is continuous and $\psi(0)=0$. For any $k \geq 2$, it follows from (3.10) and (3.11) that

$$
\ln \left(m\left(t_{k}\right)\right)-\ln \left(m\left(t_{k-1}^{+}\right)\right) \leq-r\left(t_{k}-t_{k-1}\right)
$$

and

$$
\ln \left(m\left(t_{k}^{+}\right)\right)-\ln \left(m\left(t_{k}\right)\right) \leq \ln \left(\psi\left(m\left(t_{k}\right)\right)\right)-\ln \left(m\left(t_{k}\right)\right)
$$

which imply that

$$
\begin{aligned}
& \ln \left(m\left(t_{k}^{+}\right)\right)-\ln \left(m\left(t_{k-1}^{+}\right)\right) \\
& \leq-r\left(t_{k}-t_{k-1}\right)+\ln \frac{\psi\left(m\left(t_{k}\right)\right)}{m\left(t_{k}\right)} \\
& =-r\left(t_{k}-t_{k-1}\right)+\ln \left(1+\frac{\left.2 \sqrt{m\left(t_{k}\right.}\right) g_{k}\left(\sqrt{m\left(t_{k}\right)}+K\right)+g_{k}^{2}\left(\sqrt{m\left(t_{k}\right)}+K\right.}{m\left(t_{k}\right)}\right) \\
& \leq-\xi_{k}
\end{aligned}
$$


because of assumption (iii). Thus we have

$$
m\left(t_{k}^{+}\right) \leq m\left(t_{k-1}^{+}\right) \quad \text { for } k=2,3, \ldots
$$

since $\xi_{k} \geq 0$. Let $\varepsilon>0$ be given. Since $\psi(u)$ is continuous and $\psi(0)=0$, we can find a positive number $0<\alpha<\varepsilon^{2}$ such that

$$
\psi(s)<\varepsilon^{2} \quad \text { for } s \in[0, \alpha) \text {. }
$$

Choose $\delta=\min \{\sqrt{a}, \varepsilon\}$ and let $z(t)$ be a solution of (3.5) with $0<z(0)-$ $K<\delta$. Then by $(3.10), m(t)=(z(t)-K)^{2}$ is nonincreasing in the interval $\left[t_{k-1}, t_{k}\right], k=1,2, \ldots$. Thus we have

$$
m(t) \leq \alpha \quad \text { for } t \in\left[0, t_{1}\right]
$$

and consequently

$$
m\left(t_{1}^{+}\right) \leq \psi\left(m\left(t_{1}\right)\right)<\varepsilon^{2} .
$$

This, together with (3.15), implies that $m(t) \leq \varepsilon^{2}$ for $t \geq 0$. Thus the solution $z=K$ of (3.5) is stable. By (3.14) it is easy to derive

$$
\ln \left(m\left(t_{k}^{+}\right)\right)-\ln \left(m\left(t_{1}^{+}\right)\right) \leq-\sum_{j=2}^{k} \xi_{j}
$$

Thus

$$
\lim _{k \rightarrow \infty} \ln \left(m\left(t_{k}^{+}\right)\right) \leq \ln \left(m\left(t_{1}^{+}\right)\right)-\sum_{j=2}^{\infty} \xi_{j}=-\infty
$$

which implies that $\lim _{k \rightarrow \infty} m\left(t_{k}^{+}\right)=0$. Thus we have $\lim _{t \rightarrow \infty} m(t)=0$.

In the case of $z(0)=K$, we have $z(t) \equiv K$ for all $t \geq 0$, completing the proof.

We illustrate Theorem 3.1 by the so-called logistic equation

$$
u_{i}=a \Delta u+\alpha u(K-u)
$$

(see [10]) where $K>0$ is the carrying capacity of the environment and $\alpha>0$ is the natural growth rate of the population. For this case, in condition (iii) $r$ is replaced by $\alpha K$. Therefore, increasing the growth rate, carrying capacity or the length of time between successive impulses tends to stabilise the system.

To illustrate how sharp the sufficient condition

$$
\sum_{k=1}^{\infty} \inf _{z>0}\left\{\alpha K\left(t_{k}-t_{k-1}\right)-2 \ln \left(1+\frac{g_{k}(z+K)}{z}\right)\right\}=+\infty
$$

is, we consider a special case where $t_{k}=k \tau, \tau>0$ is a constant, $g_{k}(z)=$ $\delta(z-K)$ if $z \geq K$ and $g_{k}(z)=0$ if $0 \leq z<0, \delta \in(0,1)$ is a constant. The 
above sufficient condition becomes $\alpha K \tau-2 \ln (1+\delta)>0$ i.e., $e^{\alpha K r}>(1+\delta)^{2}$. We will show that if the above inequality is not satisfied, for example, if $e^{\alpha K \tau}<1+\delta$, then the positive equilibrium point $x=K$ of the following impulsive system

$$
\left\{\begin{array}{l}
\dot{x}(t)=\alpha x(K-x), \quad t \neq k \tau, \\
x\left(k \tau^{+}\right)=x(k \tau)+g(x(k \tau)), \quad t=k \tau, k=0,1, \ldots
\end{array}\right.
$$

is not globally asymptotically stable.

In fact, let $h(z)=z+g(z)$ for $z \geq 0$, then for $k \tau<t \leq(k+1) \tau$, we have

$$
\ln \frac{x(t)}{K-x(t)}-\ln \frac{x\left(k \tau^{+}\right)}{K-x\left(k \tau^{+}\right)}=K \alpha(t-k \tau),
$$

from which we obtain

$$
x(t)=\frac{K x\left(k \tau^{+}\right)}{\left[K-x\left(k \tau^{+}\right)\right] e^{-\alpha K(t-k \tau)}+x\left(k \tau^{+}\right)}
$$

and hence

$$
x((k+1) \tau)=\frac{K h(x(k \tau))}{[K-h(x(k \tau))] e^{-\alpha K \tau}+h(x(k \tau))} .
$$

Let $y_{k}=x(k \tau)$, then we get

$$
y_{k+1}=f\left(y_{k}\right), \quad k=0,1,2, \ldots,
$$

where

$$
f(y)=\frac{K h(y)}{[K-h(y)] e^{-\alpha K \tau}+h(y)} .
$$

In the case where $y \geq K$, we have

$$
\begin{aligned}
& f(y)-y=\frac{K[(1+\delta) y-\delta K]-\left(1-e^{-\alpha K \tau}\right)[(1+\delta) y-\delta K] y-K e^{-\alpha K \tau} y}{\left(1-e^{-\alpha K \tau}\right)[(1+\delta) y-\delta K]+K e^{-\alpha K \tau}} \\
& =\frac{-(1+\delta)\left(1-e^{-\alpha K \tau}\right) y^{2}+\left[K(1+\delta)-\delta K\left(1-e^{-\alpha K \tau}\right)-K e^{-\alpha K \tau}\right] y-\delta K^{2}}{\left(1-e^{-\alpha K \tau}\right)[(1+\delta) y-\delta K]+K e^{-\alpha K \tau}} \\
& =\frac{-(1+\delta)\left(1-e^{-\alpha K \tau}\right) y^{2}+(1+\delta)\left(1-e^{-\alpha K \tau}\right) K y+\delta K y-\delta K^{2}}{\left(1-e^{-\alpha K \tau}\right)[(1+\delta) y-\delta K]+K e^{-\alpha K \tau}} \\
& =\frac{-(y-K)\left[\alpha(1+\delta)\left(1-e^{-\alpha K \tau}\right) y-\delta K\right]}{\left(1-e^{-\alpha K \tau}\right)[(1+\delta) y-\delta K]+K e^{-\alpha K \tau}}
\end{aligned}
$$

Since $e^{\alpha K \tau}<1+\delta$, we have $\delta K_{\prime}^{\prime}\left((1+\delta)\left(1-e^{-\alpha K \tau}\right)\right)>K$. Therefore, $y=\alpha \delta K /\left((1+\delta)\left(1-e^{-\alpha K \tau}\right)\right)$ is a fixed point of $f$ which means

$$
x(t) \equiv \frac{K x(0)}{[K-x(0)] e^{-\alpha K(t-K \tau)}+x(0)} \quad \text { for } t \in(k \tau,(k+1) \tau]
$$


if $x(0)=\alpha \delta K /\left((1+\delta)\left(1-e^{-\alpha K \tau}\right)\right)$. That is, the impulse creates a positive nonconstant periodic solution. Therefore, the equilibrium $x=K$ is not globally asymptotically stable.

CASE II: $c>0$. To motivate assumption (3.22) below, we consider the logistic equation

$$
u_{t}=\alpha \Delta u+u(K-u)+c .
$$

It is easy to verify that $\alpha u(K-u)+c=-\alpha(u-L)(u+M)$ where $L=$ $\frac{1}{2}\left(\sqrt{K^{2}+4 c / \alpha}+K\right)>0$ and $M=\frac{1}{2}\left(\sqrt{K^{2}+4 c / \alpha}-K\right)>0$. Therefore for $u \geq 0$

$$
(u-L)[\alpha u(K-u)+c]=-\alpha(u+M)(u-L)^{2} \leq-\alpha M(u-L)^{2} .
$$

Motivated by this observation, we make the following assumption for the general equation (3.1) with $c>0$ :

there exist constants $L>0$ and $r>0$ such that

$$
(u-K)[u f(u)+c] \leq-r(u-L)^{2} \text { for } u \geq 0 \text {. }
$$

Under this assumption, we can employ a similar argument to that for the case where $z(0)>K$ in Theorem 3.1 to prove the following result.

THEOREM 3.2. Assume that

(i) (3.22) holds, $f$ is continuously differentiable, and $\frac{d}{d u}[u f(u)]$ is uniformly bounded for $u>0$.

(ii) $g_{k}:[0, \infty) \rightarrow(-\infty,+\infty)$ is globally Lipschitz continuous and the mapping $x+g_{k}(x)$ is increasing,

(iii) $g_{k}(L)=0, k=1,2, \ldots$,

(iv) for any $z>0$,

$$
r\left(t_{k}-t_{k-1}\right)-2 \ln \left[1+\frac{g_{k}(z+L)}{z}\right] \geq \xi_{k} \geq 0, \quad k=2,3, \ldots,
$$

(v) the series $\sum_{k=1}^{\infty} \xi_{k}$ is divergent.

Then the solution $u=L$ is globally asymptotically stable. That is, for any $u_{0} \in C(\bar{\Omega},(0, \infty))$ and for any solution of $(3.1)-(3.4)$ with $c>0$, $\lim _{t \rightarrow \infty} u(t, x)=L$ for $x \in \bar{\Omega}$.

Therefore, increasing the stocking rate not only enlarges the effective carrying capacity, $L$, of the environment but also stabilises the system. Moreover, 
increasing the length between two successive moments at which impulses occur tends to stabilise the system.

\section{Acknowledgement}

Research of the first two authors was partially supported by the Natural Sciences and Engineering Research Council of Canada. Research of the last author was supported by the J. Gordon Kaplan Postdoctoral Fellowship.

\section{References}

[1] L. J. Allen, "Persistence, extinction and critical patch number for isolated populations," J. Math. Biol. 24 (1987) 617-625.

[2] D. G. Aronson, "Density dependent interaction-diffusion systems," in Proceedings of the advanced seminar on dynamics and modeling of reactive systems, Academic Press (1980).

[3] D. D. Bainov, V. Lakshmikantham and P. S. Simeonov, Theory of impulsive differential equations, Academic Press (1989).

[4] F. Brauer and D. A. Sanchez, "Constant rate population harvesting: equilibrium and stability," Theor. Pop. Biol. 8 (1975) 12-30.

[5] F. Brauer and D. A. Sanchez, "Some models for population growth with harvesting," in International conference on differential equations (H. Antosiewicz, ed.), Academic Press (1975) 53-64.

[6] F. Brauer and A. C. Soudack, "Constant-rate stocking of predator-prey systems," $J$. Math. Biol. 11 (1981) 1-14.

[7] N. F. Britton, "Aggregation and the completitive exclusion principle," J. Theor. Biol. 136 (1989) 57-66.

[8] R. S. Cantrell and G. C. Cosner, "Diffusive logistic equations with indefinite weights: population models in disrupted environments," Proc. Roy. Soc. Edin. (in press).

[9] H. I. Freedman, "Graphical stability, enrichment and pest control by a natural enemy," Math. Biosci 31 (1976) 207-225.

[10] H. I. Freedman, Deterministic mathematical models in population ecology, HIFR Consulting Ltd., Edmonton (1987).

[11] H. I. Freedman, J. B. Shukla and Y. Takeuchi, "Population diffusion in a two-patch environment," Math. Biosci. 95 (1989) 111-123.

[12] $\mathrm{H}$. I. Freedman and J. Wu, "Steady state analysis in a model for population diffusion in a multi-patch environment," preprint.

[13] J. Furter and M. Grinfeld, "Local vs. non-local interactions in population dynamics," $J$. Math. Biol. 27 (1989) 65-80.

[14] T. G. Hallam, "A temporal study of diffusion effects on a population modelled by quadratic growth," Nonlin. Anal. Th. Meth. App. 3 (1979) 123-133.

[15] G. S. Ladde, V. Lakshmikantham and A. S. Vatsala, Monotone iterative techniques for nonlinear differential equations, (Pitman, Boston, 1985).

[16] O. A. Ladyzenskaya, V. A. Solonikov and N. N. Uralawa, Linear and quasilinear equations of parabolic type, Amer. Math. Soc. Transl. Monogr. 23, Providence, 1968.

[17] V. Lakshmikantham, "Comparison results for reaction diffusion equations in Banach space," in $A$ survey of theoretical and numerical trends in nonlinear analysis, Gius. Laterza, Bari, Italy (1979) 121-156. 
[18] V. Lakshmikantham and X. Liu, "Stability criteria for impulsive differential equations in terms of two measures," J. Math. Anal. Appl. 137 (1989) 591-604.

[19] X. Liu and R. Pirapakaran, "Asymptotic stability for impulsive differential systems," $J$. Appl. Math. Comp. (in press).

[20] V.D. Millan and A. D. Myshkis, "On the stability of motion in the presence of impulses," Siber. Math. Zh. 1 (1960) 233-237 (Russian).

[21] S. G. Pandit and S. G. Deo, Differential systems involving impulses, (Springer-Verlag, New York, 1982).

[22] N. A. Perestyuk and A. M. Samoilenko, Differential equations with impulses, (Central Publishers, Kiev, USSR, 1987) (Russian).

[23] M. H. Protter and H. F. Weinberger, Maximum principles in differential equations, (Prentice-Hall, New Jersey, 1967).

[24] O. L. Smith, "The influence of environmental gradients on ecosystem stability," Amer. Nat. 116 (1980) 1-24.

[25] R. Sperb, Maximum principles and their applications, (Academic Press, New York, 1981).

[26] P. P. Zabreiko, D. D. Bainov and S. I. Kostadinov, "Stability of linear equations with impulsive effect," Tamkang J. of Math. 18 (1987) 57-63. 